\title{
Endometriosis localized to urinary bladder wall mimicking urinary bladder carcinoma
}

\author{
Mine Genç ${ }^{1}$, Berhan Genç ${ }^{2}$, Serap Karaarslan ${ }^{3}$, Aynur Solak ${ }^{2}$, Musa Saraçoğlu ${ }^{4}$ \\ ${ }^{1}$ Şifa University School of Medicine, Department of Obstetric and Gynecology, Izmir, Turkey; \\ 2 Sifa University School of Medicine, Department of Radiology, Izmir, Turkey; \\ 3 Şifa University School of Medicine, Department of Pathology, Izmir, Turkey; \\ ${ }^{4}$ Şifa University School of Medicine, Department of Urology, Izmir, Turkey.
}

\begin{abstract}
Summary Although endometriosis is a common disease in women of reproductive age, urinary system endometriosis is an exceedingly rare disease that may cause important clinical problems. In this paper we discussed a 42-year-old woman who had urinary bladder endometriosis misdiagnosed as urinary bladder tumor in imaging modalities. The diagnosis of endometriosis was made by histopathological examination of the operative material after partial resection of the urinary bladder. Urinary bladder endometriosis causes nonspecific signs and symptoms in many patients. In female patients presenting with unexplained urinary symptoms the differential diagnosis should include urinary bladder endometriosis that may mimic urinary bladder cancer and lead to difficulties in making definitive preoperative diagnosis.
\end{abstract}

KEY WORDS: Endometriosis; Urinary bladder; Urinary tract endometriosis.

Submitted 22 December 2013; Accepted 31 March 2014

\section{INTRODUCTION}

Endometriosis is the presence of functional endometrial tissue in ectopic foci outside the uterine cavity. Approximately $10 \%$ of women of reproductive age suffer from endometriosis (1). Urinary tract endometriosis (UTE) is observed in 1-2\% of women with endometriosis. Among women with UTE, about $80 \%$ have urinary bladder involvement (2). We presented herein a patient presenting to our clinic with pelvic pain and dysuria who was diagnosed with an endometriosis focus on the roof of the urinary bladder, which was initially misdiagnosed as primary urinary bladder cancer.

\section{Case report}

A 42-year-old woman, who had two previous deliveries with caesarean section, presented with pelvic pain and dysuria worsening in the last 6 months. She had no hematuria. Her menstrual cycles were regular; however, she stated that the pelvic pain and dysuria worsened during menstrual period. The gynecological examination was not remarkable. Transabdominal ultrasonographic examination revealed an irregular contoured, solid, hypoechoic lesion of $4 \times 1.5 \mathrm{~cm}$ on the roof of the urinary bladder. Magnetic resonance imaging showed a mass lesion with homogenous contrast uptake that appeared hypointense in T1W and hyper-intense in T2W (Figure 1). MRI images suggested a malignant diagnosis. Flexible cystoscopy was performed and a biopsy sample was taken from the lesion. Biopsy result was reported as nonspecific urinary bladder tissue. The tumoral mass was then removed with laparotomy and partial cystectomy. Examination of the excised tumoral tissue showed macroscopic hemorrhagic foci on the urinary bladder wall. Microscopic examination revealed some areas consistent with cystitis glandularis and, in addition, other areas consistent with endometrial glands and stroma inside the muscular layer (Figure 2).

\section{Discussion}

Endometriosis is the presence of functional endometrial tissue in ectopic foci outside the uterine cavity. Urinary tract endometriosis (UTE) is observed in 1-2\% of women with endometriosis. Among women with UTE, about 80\% have urinary bladder involvement (2). This is followed by, in descending order of frequency, ureters, kidney, and urethra. Endometrial lesions may assume the appearance of a polypoid mass similar to that of urinary bladder carcinoma when they grow towards the mucosa alongside the muscle layers. Urinary bladder endometriosis is termed as "primary" or "secondary" depending on its type of onset: the primary urinary bladder endometriosis occurs when the endometrial tissue is congenitally located within the urinary bladder wall. The secondary urinary bladder endometriosis, on the other hand, is a iatrogenic lesion occurring in patients delivering a baby with caesarean section or undergoing a pelvic operation such as hysterectomy. Up to $50 \%$ of patients with urinary bladder endometriosis have a previous history of a pelvic operation. Symptoms of urinary bladder endometriosis may vary depending on the localization and the site of the lesion. The symptoms may include recurrent cystitis, pelvic pain, dysuria, tenesmus, and burning sensation. Hematuria is present in 20-35\% of cases. Menouria (hematuria with

\footnotetext{
No conflict of interest declared.
} 
menstruation) is not as common as the acute urethral syndrome, and it is seen in only $20-25 \%$ of cases when the mucosa is affected. Negative urinary cultures in symptomatic individuals at premenopausal period may suggest urinary bladder endometriosis. Ultrasonography (USG) is the first step in the diagnosis of urinary bladder endometriosis. USG may provide information with respect to lesion size and localization as well as the degree of infiltration of mucosa and detrusor muscle. Magnetic resonance imaging (MRI) is an excellent modality for demonstrating urinary bladder endometriosis (3). The role of cystoscopy is limited in diagnosing urinary bladder endometriosis since the lesion of urinary bladder endometriosis is usually located on the serosal surface or in the submucosal layer of the urinary bladder. Cystoscopic findings may be normal despite a positive transvaginal ultreasonography (TV
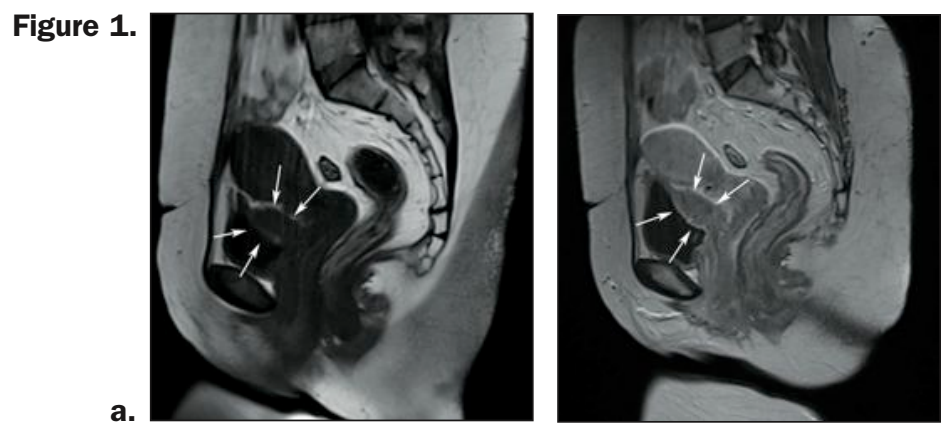

Sagittal T1-weighted images: the pre-contrast image (a) demonstrates a mass grown within the muscle layer that shows no luminal projection at the posterior part of the urinary bladder roof (arrows). Post-contrast image (b) demonstrates that the mass has a diffuse homogenous contrast uptake (arrows).

Figure 2.

a.
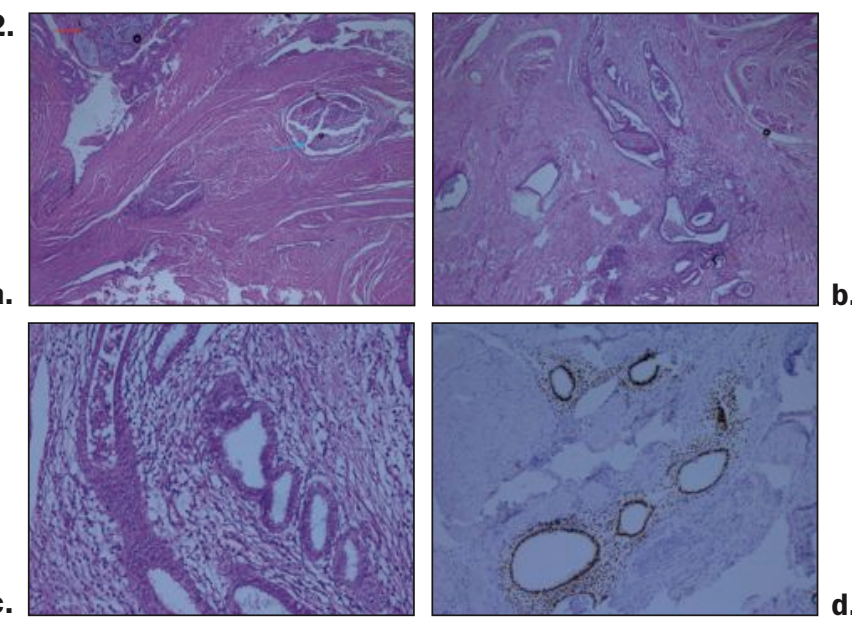

(a) At the upper part of the image there are areas with features of cystitis glandularis at the urinary bladder's urothelial epithelium (red arrow) and areas of endometriosis (green arrow) composed of foci of endometrial glands and stroma within muscularis propria at the submucosal layer (H\&E x 10); (b) Foci of endometriosis within the muscularis propria of the urinary bladder (H\&E x 10); (c) A closer view of the areas of endometriosis (H\&E x 40); and (d) estrogen receptor (ER) positivity in the foci of endometriosis (ER $x 20)$.
USG). Endoscopic biopsy is of pivotal importance for differentiating the lesions from carcinomas, varices, papillomas, angiomas, and also detrusor mesenchymal tumors. However, cystoscopic biopsies except for transurethral resection (TUR) procedures are not always diagnostic. Also in our case, the lesion went unnoticed in cystoscopy as it was localized to submucosal and intramuscular regions.

The differential diagnosis of urinary bladder endometriosis include epithelial tumors of urinary bladder, hemangiomas, myomas of anterior uterine wall, detrusor muscle leiomyomas, fibromas, glandular cystitis, nephrogenic adenoma, and diverticulitis.

Treatment of disease varies by certain factors including age, fertility preferences, disease extension, severity of lower urinary system symptoms, presence of other pelvic lesions, and degree of menstrual dysfunction. The therapy may be in the form of medical therapy (hormonal agents), surgery, or a combination of the two. In young women willing to maintain their fertility gonadotropin releasing hormone $(\mathrm{GnRH})$ agonists and antagonists, progestins, danazol, and combined oral contraceptives are used. Surgical therapy consists of transurethral surgery (TUR) and partial cystectomy (laparotomic or laparoscopic).

In conclusion, the urinary system is the second most commonly involved site by extrapelvic endometriosis. It constitutes $1-2 \%$ of all endometriosis cases. Fifty percent of patients with urinary bladder endometriosis have a previous history of a pelvic operation (including caesarean section). The diagnosis may be done by ultrasonography (USG), MRI, or cystoscopy. The therapy may be in the form of medical therapy (hormonal agents), surgery, or a combination of the two. The diagnosis of endometriosis should definitely be remembered in patients in whom USG detects a mass at the urinary bladder wall but cystoscopy fails to show any lesion.

\section{REFERENCES}

1. Olive DL, Schwartz LB. Endometriosis. N Engl J Med. 1993; 328:1759-69.

2. Shook TE, Nyberg LM. Endometriosis of the urinary tract. Urology 1988; 31:1-6.

3. Beaty SD, Silva AC, de Petris G. Bladder endometriosis:multrasound and MRI findings. Radiology Case Reports. 2006; 1:92-95.

\section{Correspondence}

Mine Genç, MD (Corresponding Author)

doktorminegenc@gmail.com

Sifa University School of Medicine,

Department of Obstetric and Gynecology

Fevzipasa Boulevard, N: 172/2 Basmane 35240 Izmir, Turkey

d.

Berhan Genç, MD

Aynur Solak, MD

Sifa University School of Medicine, Department of Radiology Izmir, Turkey

Serap Karaarslan, MD

Sifa University School of Medicine, Department of Pathology Izmir, Turkey

Musa Saraçoglu, MD

Sifa University School of Medicine, Department of Urology Izmir, Turkey 